from TNF- $\alpha$ and IL-6, depending on the degree of weight loss was not detected.

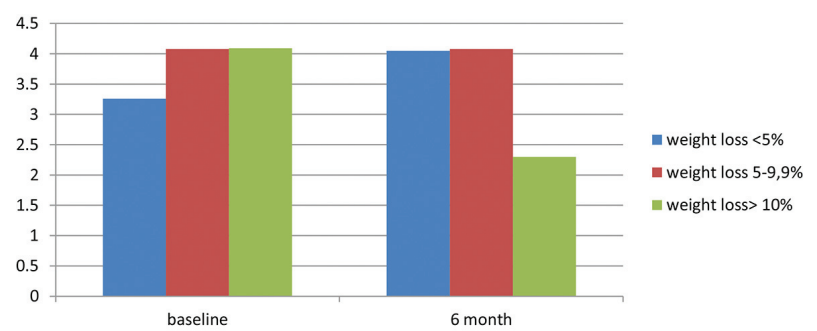

Abstract THU0457 - Figure 1. Dynamics of CRP depending on the degree of weight loss $(p<0.05)$

Conclusion: The results of the study demonstrated a significant effectiveness of the complex treatment of obesity in patients with knee OA, compared with the use of only non-pharmacological methods of weight loss. A decrease in body mass of more than $5 \%$ helps to improve the clinical manifestations of knee $\mathrm{OA}$ and the quality of life of patients. A decrease in body weight of more than $10 \%$ demonstrates a decrease in the level of CRP, which suggests an effect on meta-inflammation in OA.

Disclosure of Interests: Ekaterina Strebkova: None declared, Ludmila Alekseeva Speakers bureau: Bayer, Boeringer-ingelheim, Gedeon-Richter, Servier DOI: 10.1136/annrheumdis-2019-eular.6793

\section{THU0458 EFFICACY AND SAFETY FROM A PHASE 2B TRIAL OF SM04690, A NOVEL INTRA-ARTICULAR WNT PATHWAY INHIBITOR FOR THE TREATMENT OF OSTEOARTHRITIS OF THE KNEE}

Yusuf Yazici ${ }^{1}$, Timothy Mcalindon ${ }^{2}$, Allan Gibofsky ${ }^{3}$, Nancy Lane ${ }^{4}$

Christian Lattermann $^{5}$, Nebojsa Skrepnik ${ }^{6}$, Christopher Swearingen ${ }^{1}$,

Anita Difrancesco ${ }^{1}$, Jeyanesh Tambiah ${ }^{1}$, Marc Hochberg ${ }^{7}$. 'Samumed, LLC, San

Diego, United States of America; ${ }^{2}$ Tufts Medical Center, Boston, United States of

America; ${ }^{3}$ Weill Cornell Medical College, New York, United States of America; ${ }^{4}$ UC Davis Medical Center, Davis, United States of America; ${ }^{5}$ Brigham and Women's Hospital, Boston, United States of America; ${ }^{6}$ Tucson Orthopedic Institute, Tucson, United States of America; ${ }^{7}$ University of Maryland School of Medicine, Baltimore, United States of America

Background: A phase 2a study of SM04690, a small-molecule, intra-articular (IA) Wnt pathway inhibitor reduced knee pain and improved physical function and medial joint space width (mJSW) at 52 weeks in subgroups of subjects with unilateral symptomatic knee osteoarthritis (OA) compared to placebo $(\mathrm{PBO}){ }^{1}$

Objectives: A 24-week phase $2 \mathrm{~b}$ study was conducted to refine patientreported outcome (PRO) measures, target population, medication dose, and to evaluate safety. PRO results for Weeks 12 and 24 are presented here.

Methods: Study subject inclusion criteria required ACR-defined knee OA, Kellgren-Lawrence (KL) grades 2-3, and Pain Numeric Rating Scale (NRS) $\geq 4$ and $\leq 8$ in the target knee and $<4$ in the contralateral knee. A single IA injection of $2 \mathrm{~mL} S M 04690(0.03,0.07,0.15$, or $0.23 \mathrm{mg})$, vehicle $\mathrm{PBO}$, or sham (dry needle only) was given in the target knee at baseline. PRO endpoints included change from baseline in weekly average of daily pain in the target knee by NRS diary (NRS) [0-10], Western Ontario and McMaster Universities Osteoarthritis Index (WOMAC) Pain [0-100], WOMAC Physical Function [0-100], and Patient Global Assessment (PtGA) [0-100]. Differences between active treatment groups and vehicle PBO were analyzed with baseline-adjusted analysis of covariance (ANCOVA).

Results: 695 subjects (mean age 59.0 [ \pm 8.5$]$ years, BMI 29.0 [ \pm 4.0$]$ kg/ $\mathrm{m}^{2}$, female $58.4 \%$, KL3 57.3\%) were enrolled and dosed; 635 subjects (91.4\%) completed the study. No meaningful differences in the incidence of adverse events were observed between treatment and control groups. In the Full Analysis Set, significant improvements from baseline compared to vehicle PBO were observed in pain NRS for $0.07 \mathrm{mg}$ (Week 12 $[P=0.001]$, Week $24[P=0.031]$ ) and $0.23 \mathrm{mg}$ (Week $12[P=0.012]$, Week $24[P=0.022])$ SM04690 dose groups (Figure). Similar improvements were observed in WOMAC Pain for $0.07 \mathrm{mg}$ (Week $12[P=0.04]$ ) and $0.23 \mathrm{mg}$ (Week $12[P=0.003]$, Week $24[P=0.031]$ ) dose groups. For WOMAC Physical Function, improvements were observed for $0.07 \mathrm{mg}$ (Week 12 $[p=0.021])$ and $0.23 \mathrm{mg}$ (Week $12[p=0.006]$, Week $24[P=0.017])$ dose groups. PtGA improvements were observed for $0.07 \mathrm{mg}$ (Week 12
$[P=0.031])$ and $0.23 \mathrm{mg}$ (Week $12[P=0.010]$, Week $24[P=0.033])$ dose groups.

Conclusion: SM04690, in development as a potential disease-modifying OA drug, showed in this phase $2 b$ study statistically significant improvements from baseline in both the $0.07 \mathrm{mg}$ and $0.23 \mathrm{mg}$ dose groups compared to vehicle $\mathrm{PBO}$ for Pain NRS, WOMAC Pain, WOMAC Physical Function, and PtGA. These data support the continued development of SM04690 as a treatment for knee OA. Phase 3 studies are being planned.

\section{REFERENCE:}

[1] Yazici Y, et al. Arthritis Rheumatol. 2017; 69 (suppl 10).
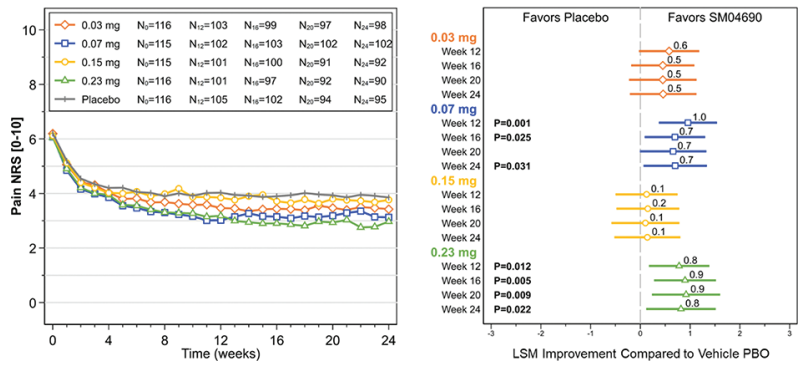

Figure. Actual observations over time and ladder plots depicting least squares mean (LSM) improvement of Pain NRS $( \pm 95 \% \mathrm{Cl}$ ) in SM04690 compared to vehicle PBO, adjusted for baseline.

Disclosure of Interests: Yusuf Yazici Shareholder of: Samumed, LLC Consultant for: Celgene Corporation, BMS, Genentech, Sanofi, Employee of: Samumed, LLC, Timothy McAlindon Grant/research support from: Samumed, LLC, Consultant for: Samumed, LLC, Astellas, Flexion, Pfizer, Regeneron,

Seikugaku, Allan Gibofsky Shareholder of: AbbVie, Amgen, Johnson\&Johnson, Regeneron, Consultant for: AbbVie, Pfizer, Horizon, Celgene, Novartis/Sandoz, Samumed, LLC, Merck, Flexion, Lilly, Speakers bureau: AbbVie, Celgene, Pfizer, Flexion, Lilly, Merck, Nancy Lane Consultant for: Samumed, LLC, Christian Lattermann Shareholder of: Cocoon, Consultant for: Samumed, LLC, Vericel, Cariheal, Joint Restoration Foundation, Nebojsa Skrepnik Grant/research support from: Samumed, LLC, Consultant for: Orthofix, Sanofi, Regeneron, Christopher Swearingen Shareholder of: Samumed, LLC, Employee of: Samumed, LLC, Anita DiFrancesco Shareholder of: Samumed, LLC, Employee of: Samumed, LLC, Jeyanesh Tambiah Shareholder of: Samumed, LLC, Employee of: Samumed, LLC, Marc Hochberg Shareholder of: BriOri Biotech, Theralogix LLC., Consultant for: Bristol Myers Squibb, Eli Lilly, EMD Serono, Novartis Pharma AG, Pfizer Inc., Samumed LLC, Symic Bio Inc., Theralogix LLC, TissueGene Inc. TLC Biopharmaceuticals, Inc., Zynerba, Galapagos, IQVIA, Hoffman LaRoche.

DOI: 10.1136/annrheumdis-2019-eular.5045

\section{THU0459 PREDICTORS ASSOCIATED WITH RAPID PROGRESSION OF KNEE OSTEOARTHRITIS}

Elena Taskina ${ }^{1}$, Ludmila Alekseeva ${ }^{1}$, Natalia Kashevarova ${ }^{1}$, Sergey Anikin ${ }^{1}$, Evgenia Sharapova ${ }^{1}$, Ekaterina Strebkova ${ }^{1}$, Lena Zonova ${ }^{2}$, Tatiana Raskina ${ }^{3}$, Elvira Otteva ${ }^{4}$, Irina Vinogradova ${ }^{5}$, Aleksandr Lila ${ }^{1}{ }^{1}$ V. A. Nasonova Research Institute of Rheumatology, Moscow, Russian Federation; ${ }^{2}$ FSBEI HE Novosibirsk State Medical University, Novosibirsk, Russian Federation; ${ }^{3}$ SBEI HE KemSMU $\mathrm{MOH}$, Kemerovo, Russian Federation; ${ }^{4}$ Regional SBHI CDC MOH Khabarovsky kray, Khabarovsk, Russian Federation; ${ }^{5}$ SHI Ulyanovsk regional affiliated hospital, Ulyanovsk, Russian Federation

Objectives: To identify knee OA rapid progression factors in a multicenter prospective study

Methods: 185 female patients from 5 RF constituent territories aged 4075 with confirmed Kellgren-Lawrence score grade I-III knee OA were included into prospective study after signing the informed consent form Mean age was $59,2 \pm 7,4$ years (42-75), mean BMl-27,7 $\pm 4,4 \mathrm{~kg} / \mathrm{m}^{2}$, mean disease duration-12 $\pm 8,1$ years. Individual patient's medical record included relevant anthropometric data, records from case history and clinical examination, VAS articular pain assessment, WOMAC scores, comorbidities. Instrumental diagnostic methods included plain radiography of knee joints, DEXA of the lumbar spine and femoral neck, MRI examination of knee joints. Stage II knee OA was established in 135 (73\%) out of 185 patients, stage III - in 50 (27\%). 